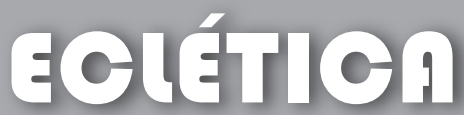 química
}

\section{VIABILIDADE DO EMPREGO DE ÁCIDO FLUORÍDRICO $10 \%$ PARA A REMOÇÃO DE ÍONS PARAMAGNÉTICOS EM AMOSTRAS DE SOLOS E SUAS RESPECTIVAS FRAÇÕES FÍSICAS}

\author{
Larissa Macedo dos Santos ${ }^{1,2}$, Marcelo Luiz Simões ${ }^{1}$, Célia Regina Montes ${ }^{3}$, Adolpho Jose Melfí, Ladislau \\ Martin-Neto ${ }^{1}$ \\ ${ }^{1}$ Embrapa Instrumentação Agropecuária; ${ }^{2}$ Universidade de São Paulo, Instituto de Química de São Carlos; ${ }^{3}$ Universidade de São Paulo, \\ Centro de Energia Nuclear na Agricultura; ${ }^{4}$ Universidade de São Paulo, Escola Superior de Agricultura Luiz de Queiroz. \\ Autor Correspondente - Larissa Macedo dos Santos \\ Embrapa Instrumentação Agropecuária - Rua XV de Novembro, 1452 \\ 13560-970 - São Carlos - SP - Brasil \\ Caixa Postal - 741 \\ Telefone: 16-3374-2477, Fax: 16-3372-5958 \\ e-mail: larissa@cnpdia.embrapa.br
}

\begin{abstract}
RESUMO: A utilização de efluentes de esgoto tratado na irrigação, ao invés de realizar a disposição deste subproduto nos cursos d’água tem sido uma alternativa antiga, popular e atrativa, com triplo propósito: tratamento complementar do efluente, fonte de água e de nutrientes ao sistema solo-planta. Entretanto, no Brasil, há falta de tradição na reciclagem de águas residuárias, particularmente, do efluente de esgoto tratado e, conseqüentemente, poucos têm sido as pesquisas relacionadas a este tema. Com a finalidade de fornecer mais informações para subsidiar as pesquisas nesta área, este trabalho visa avaliar, por meio de técnicas químicas e espectroscopias, o processo de humificação na matéria orgânica dos solos irrigados com efluente de esgoto tratado em experimentos de campo. Os cinco tratamentos estudados foram: TSI (controle) - irrigado com água potável e adição de nitrogênio via fertilizante mineral, T100, T125, T150 e T200 irrigado com efluente de esgoto tratado e adição de nitrogênio via fertilizante mineral. Os resultados obtidos mostram variações no teor de carbono e no grau de humificação, para os solos irrigados com efluente de esgoto tratado, decorrentes do aumento da atividade de decomposição da matéria orgânica, estimulada pelo aumento de água no solo.
\end{abstract}

Palavras-Chave: Matéria Orgânica do Solo, Efluente de Esgoto Tratado, Técnicas Químicas e Espectroscópicas

\section{INTRODUÇÃO}

O tratamento de esgoto mediante o emprego de lagoas de estabilização, amplamente utilizado nas cidades de pequeno a médio porte, sobretudo, no interior do Estado de São Paulo, gera dois subprodutos: (i) o lodo de esgoto, e (ii) o efluente de esgoto tratado.

O efluente de esgoto tratado é um tipo de água residuária amplamente empregado na irrigação de culturas em países como Austrália e Israel [1]. Essa água residuária difere da convencionalmente empregada na irrigação por apresentar con- 
centrações variáveis de compostos como, matéria orgânica, nutrientes (principalmente nitrogênio), sais (incluindo sais de sódio), demanda química de oxigênio, demanda bioquímica de oxigênio e patógenos, dependendo da natureza do esgoto bruto e do tipo de tratamento empregado [2]

A matéria orgânica do solo é um componente importante dos agroecossistemas por interferir tanto na qualidade do solo, e, por conseguinte, na produtividade das culturas, como na sustentabilidade ambiental [3].

Em solos tropicais e subtropicais altamente intemperizados, a matéria orgânica tem grande importância no fornecimento de nutrientes às culturas, na retenção de cátions, na complexação de elementos tóxicos e de micronutrientes, na esde água, na aeração e na atividade e diversidade de água, na aeração e na atividade e diversidade
microbiana, constituindo assim, um componente microbiana, constituindo assim, um componente
fundamental para a capacidade produtiva do solo [4].

Nos últimos anos, a aplicação de espectroscopias como: ressonância magnética nuclear, ressonância paramagnética eletrônica, fluorescência de luz UV-visível e fluorescência induzida por laser vêm demonstrando grande potencial para laser vên devisio auxiliar no en processos relacioContudo, a composição mineralógica dos solos tropicais pode dificultar as análises por ressonância magnética nuclear de ${ }^{13} \mathrm{C}$ no estado sólido e por ressonância paramagnética eletrônica. Solos com altos teores de $\mathrm{Fe}^{3+}$, como os latossolos e argissolos, são de grande ocorrência no Brasil. $\mathrm{O}$ íon $\mathrm{Fe}^{3+}$ dos óxidos interfere nos espectros da ressonância magnética nuclear de ${ }^{13} \mathrm{C}$ no estado sólido e da ressonância paramagnética eletrônica.

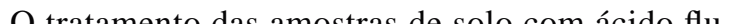
oŕdrico é uma estrégia para rolo comer esse ín orídrico é uma estratégia para remover esse ion da
amostra [10] e, assim, permitir a utilização dessas amostra [10] e, assi
espectroscopias.

\section{MATERIAIS E MÉTODOS}

\section{Área experimenta}

O campo experimental está localizado no município de Lins-SP em área anexa a estação de esgoto municipal.

\section{Classificação do solo}

O solo é classificado como Agissolo Vermelho distrófico latossólico de textura média argilosa [11].

EET

O EET foi obtido por meio do sistema de lagoa de estabilização (Estação de Tratamento de Esgoto Municipal, Lins-SP).

\section{Tratamento}

200: irrigação com EET e umidade do solo 100\% acima da capacidade de campo.

\section{Coleta das amostras de solo}

As amostras foram coletas em maio de 2007, um ano e dois meses após o plantio da cana de açúcar sob irrigação com EET.

\section{Amostragem}

As amostras de solo foram coletadas aleatoreamente nas camadas superficiais (0-10 e 10-20 $\mathrm{cm})$ e subsuperficiais $(20-40,40-60,60-80$ e 80 $100 \mathrm{~cm}$ ) nas parcelas experimentais.
O fracionamento físico dos complexos organominerais foi realizado por peneiramento e seganominerais foi realizado por peneiramento e senas frações $>53 \mu \mathrm{m}$ (areia), 20-53 $\mu \mathrm{m}$ (silte grosso), 2-20 $\mu$ m (silte fino) e $<2 \mu \mathrm{m}$ (argila)

\section{Tratamento com ácido fluorídrico}

As amostras compostas de solo foram tratadas com 8 lavagens de solução de ácido fluoridrico $10 \%$, segundo metodologia descrita na literatura [10].

\section{Teor de cinzas}

O teor de cinzas foi obtido por calcinação em cadinhos de platina: 10-100 mg de solo livre de umidade, a $600^{\circ} \mathrm{C}$ em mufla, durante 2 horas. O teor de cinzas foi calculado por diferença de teor

Tabela 1. Teor de cinzas das amostras de solo e frações argila e areia, antes e após as lavagens com soluções de ácido fluorídrico $10 \%$

\begin{tabular}{cccc}
\hline Amostras & Tratamento* $^{*}$ & Profundidade $(\mathrm{cm})$ & Teor de cinzas (\%) \\
(antes da lavagem com HF 10\%) \\
solo & 200 & $0-10$ & 96,3 \\
fração areia & 200 & $0-10$ & 97,9 \\
fração argila & 200 & $0-10$ & 77,1 \\
\hline
\end{tabular}

*Tratamento: irrigação com EET e umidade do solo 100\% acima da capacidade de campo, denominado 200.

Na Figura 1 são mostrados os espectros de ressonância paramagnética eletrônica obtidos para a fração argila antes e após as lavagens com solução de ácido fluorídrico $10 \%$. 
(A) Antes do tratamento com HF

(B) Após tratamento com HF

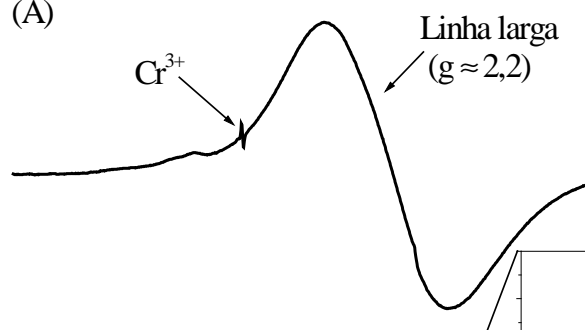

(B)

ROO

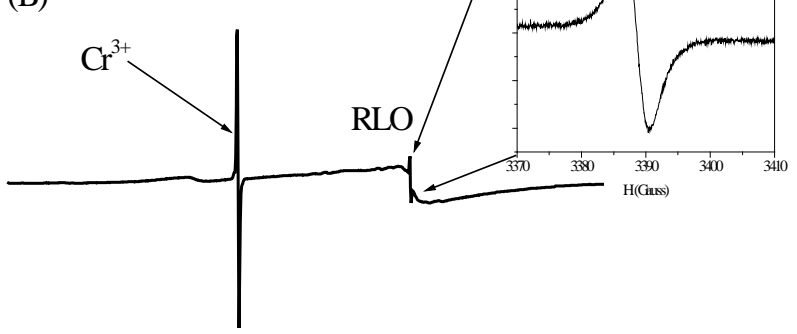

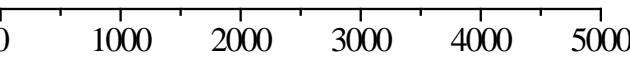

$\mathrm{H}$ (Gauss)

Figura 1. Espectros de ressonância paramagnética eletrônica obtidos para a fração argila antes e após as

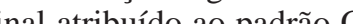
larga em $\mathrm{g} \approx 2,2$ atribuído a interação dipolar $\mathrm{Fe}^{3+} \mathrm{e}$ o sinal do radical livre orgânico (RLO).

O espectro obtido para a fração argila sem fluorídrico (Figura 2 e 3) não foi observado altetratamento com ácido fluorídrico (Figura 1) é ca- ração significativa do sinal característico de íons provavelmente, ao alto teor de areia $(\approx 80 \%)$ do ais ferromag- solo estudado fato este que pode influenciar signeticos. Contudo, não se exclui também a possibi- nificativamente na dissolução de silicatos, visto idade de alargamento espectral associado com o que a fração areia é composta principalmente por aumento da taxa de relaxação por interação dipo- quartzo, o qual é característico por apresentar uma lar [14]. O alargamento espectral observado invia- alta resistência ao tratamento com ácido fluorídribiliza a detecção do sinal do radical livre orgânico e, assim, obter informações sobre a matéria orgâica. No entanto, no espectro obtido para a fração rgila após o tratamento com ácido fluorídrico (Fi1) foi observada significaiva dinir sina caraístco dé́ . ça ção de ácido fluorídrico $10 \%$ para a fração argila. Contudo, nos espectros obtidos para as amostras de solo e fração areia após as lavagens com ácido
Na Figura 1 é mostrado o espectro obtido para o radical livre orgânico que apesar de pouco intenso, confirma a melhora espectral obtida após tratamento com solução de ácido fluorídrico

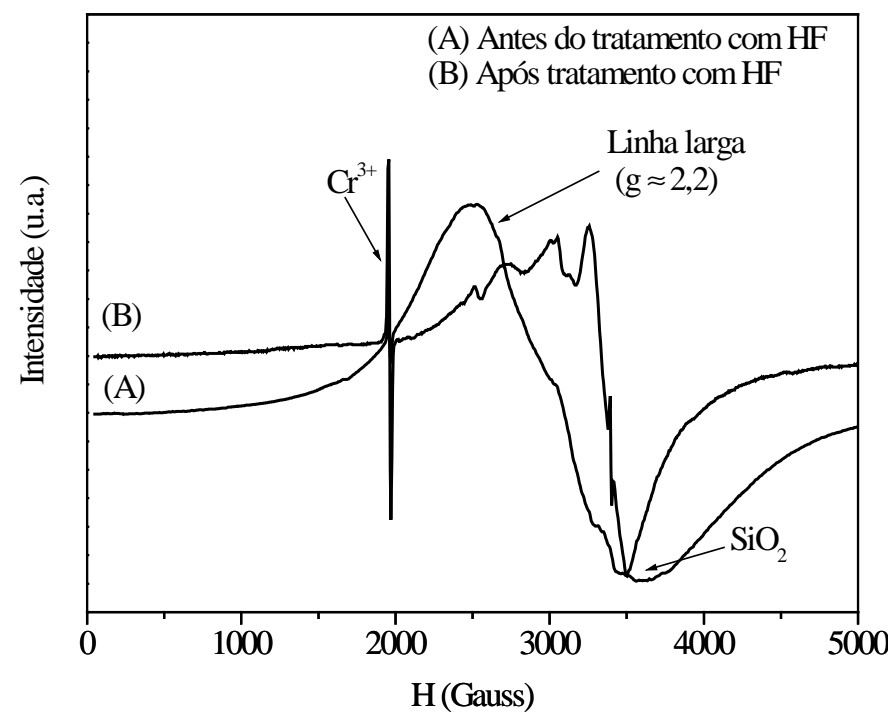

Figura 2. Espectros de ressonância paramagnética eletrônica obtidos para o solo antes e após as lavagens com solução de ácido fluorídrico $10 \%$. Detalhe mostrando o sinal atribuído ao padrão $\mathrm{Cr}^{3+}$, linha larga em $\approx 2,2$ atribuído a interação dipolar $\mathrm{Fe}^{3+} \mathrm{e}$ o sinal atribuído ao quartzo $\mathrm{SiO}_{2}$

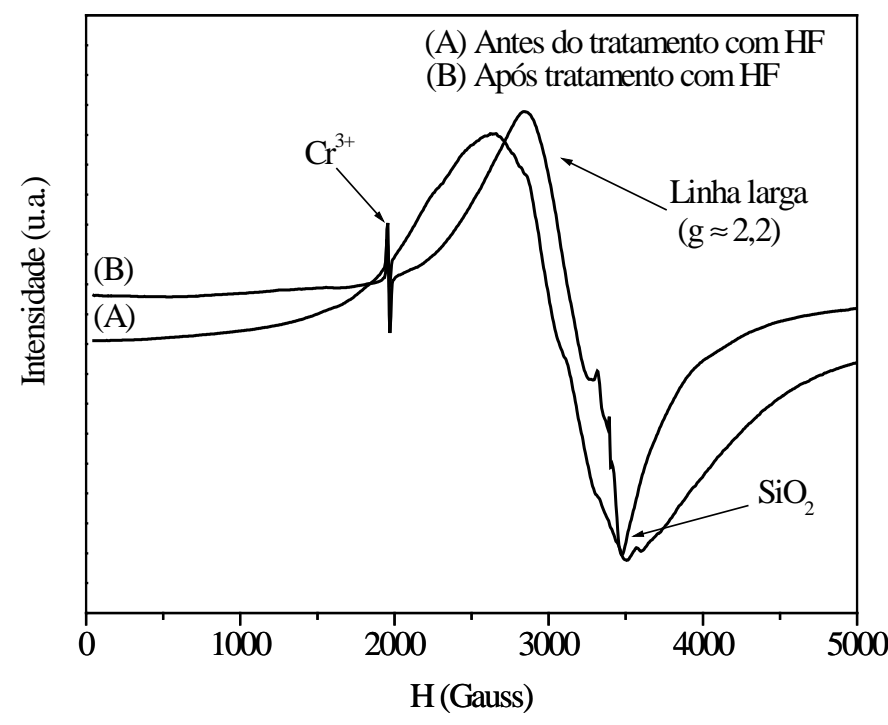

Figura 3. Espectros de ressonância parmagnética eletrônica obtidos para a fração areia antes e após as lavagens com solução de ácido fluorídrico $10 \%$. Detalhe mostrando o sinal atribuído ao padrão $\mathrm{Cr}^{3+}$, linha larga em $\mathrm{g} \approx 2,2$ atribuído a interação dipolar $\mathrm{Fe}^{3+} \mathrm{e}$ o sinal atribuído ao quartzo $\mathrm{SiO}_{2}$ 


\title{
CONCLUSÕES
}

Considerando os resultados obtidos, concluimos que é possível a utilização da ressonância paramagnética eletrônica para avaliar a dinâmica da matéria orgânica de solos, com altos teores de íons paramagnéticos $\mathrm{Fe}^{3+}$, irrigados com EET, a partir do estudo da fração argila, onde geralmente, encontra-se o maior percentual de matéria orgânica dos solos. Visto que, os resultados evidenciam a melhora espectral para a fração argila após o tratamento com solução de ácido fluorídrico $10 \%$.

\section{AGRADECIMENTOS}

À Embrapa Instrumentação Agropecuária pela infra-estrutura cedida. À FAPESP, CNPq e CAPES pelo auxílio concedido.

\begin{abstract}
Treated sewage effluent utilization for irrigation represents an antique, popular and attractive alternative to the common disposal of effluent to watercourses and includes three main purposes: effluent complementary treatment, water and nutrient source to the soil-plant system. However, because in Brazil no experiences in wastewater recycling exists consequently few scientific studies were carried out despite the importance of the subject. The present study aimed to evaluate by chemical and spectroscopy techniques the soil organic matter from soils irrigated with treated sewage effluent. Five treatments were evaluated: TSI (control) irrigation with potable water and addition of the nitrogen as mineral fertilizer; T100, T125, T150 and T200 - irrigation with treated sewage sludge effluent and addition of the nitrogen as mineral fertilizer. The results obtained shown changes in the carbon contend and humification degree for the soils irrigated with treated sewage sludge effluent due to the increase activity of the decomposition of organic matter, stimulated by the increase of water in the soil.
\end{abstract}

\section{REFERÊNCIAS BIBLIOGRÁFICAS}

[1] M. Edraki, H.B. So, E.A. Gardner. Agricultural Water Management, 67 (2004) 157.

[2] A. Feigin, I. Ravina, J. Shalhevet. Irrigation with treated sewage effluent: management for environmental protection: Berlin, Springer-Verlag, 1991. 224 p.

[3] J. Diekow, L. Martin-Neto, D.M.B.P. Milori, P.C. Conceição, C. Bayer, J. Mielniczuk. Sistemas de preparo do solo e características espectroscópicas da matéria orgânica em ambientes tropicais e subtropicais brasileiros: São Carlos, Embrapa Instrumentação Agropecuária.-Boletim de Pesquisa e Desenvolvimento, 2005. 16 p.

[4] F.J. Stevenson. Humus Chemistry: genesis, composition, reactions: New York, John Wiley, 1994. 496 p.

[5] A. Jouraiphy, S. Amir, M.E. Gharous, J-C. Revel, M. Hafidi. International Biodeterioration \& Biodegradation, 56 (2005) 101.

[6] J. Pajaczkowska, A. Sulkowska, W.W. Sulkowski, M. Jedrzejczuk. Journal of Molecular Structure, 651-653 (2003) 141.
[7] J. Polak, W.W. Sulkowski, W.P. Bartoszek. Journal of Molecular Structure, 744-747 (2005) 983.

[8] M. González-Pérez, L. Martin-Neto, S.C. Saab, E.H. Novotny, D.M.B.P. Milori, V.S. Bagnato, L.A. Colnago, W.J. Melo, H. Knicker. Geoderma, 118 (2004) 181.

[9] L.M. Santos, M.L. Simões, W.T.L. Silva, D.M.B.P. Milori, C.R. Montes, A.J. Melfi, L. Martin-Neto. Eclética Química, 34 (2009) 39.

[10] C.N. Gonçalves, R.S.D. Dalmolin, D.P. Dick, H. Knicker, E. Klamt, I. Kögel-Knabner. Geoderma, 116 (2003) 373.

[11] Empresa Brasileira de Pesquisa Agropecuária. Centro Nacional de Pesquisa de Solos. Sistema Brasileiro de Classificação de Solos: Rio de Janeiro, 1999. 412 p.

[12] C.B. Tanner, M.L. Jackson. Soil Science Society Proceedings, 60-65 (1947).

[13] L. Martin-Neto, O.R. Nascimento, J. Talamoni, N.R. Poppi. Soil Science, 151 (1991) 369.

[14] E.H. Novotny, L. Martin-Neto. Geoderma, 106 (2002) 305. 